\title{
Fiber Endoscopes Utilizing Liquid Tunable-Focus Microlenses Actuated Through Infrared Light
}

\author{
Xuefeng Zeng, Carter T. Smith, Jon C. Gould, Charles P. Heise, and Hongrui Jiang, Senior Member, IEEE
}

\begin{abstract}
We report on prototype fiber endoscopes with tunable-focus liquid microlenses integrated at their distal ends and actuated through infrared (IR) light. Tunable-focus microlenses allow minimal back-and-forth movements of the scopes themselves and different depths of focus (DOFs), thus having spatial depth perception in the obtained images. The liquidmicrolens was formed by a water-oil meniscus pinned at a hydrophobichydrophilic boundary at an aperture. IR light-responsive hydrogel microstructures were formed by photopatterning thermo-responsive $\mathrm{N}$-isopropylacrylamide hydrogel with entrapped IR light absorbing gold nanoparticles. The volumetric change in the hydrogel microstructures regulated the pressure difference across the water-oil interface and thus varied its focal length. The operations of the microlenses were realized through light transmitted via optical fibers. The images obtained from the microlenses were transferred via image fiber bundles. For two alignments between the hydrogel structures and the fibers, the response times of the microlenses are 65 and $20 \mathrm{~s}$, respectively. Images of the simulated polyps in simulated colons were obtained. The range of focal length of a typical microlens was from 52 to $8 \mathrm{~mm}$. The angle of view of an endoscope was from $77^{\circ}$ to $128^{\circ}$. A microlens array could potentially be utilized to simultaneously obtain different DOFs and to increase the field of view.

[2010-0278]
\end{abstract}

Index Terms-Endoscopes, hydrogel, liquid-liquid meniscus, nanoparticles, optical fibers, tunable-focus microlenses.

\section{INTRODUCTION}

$\mathbf{E}$ NDOSCOPY, which was intended as a methodology to obtain images of the interior surfaces of cavities in human bodies, can be traced back to 1800 [1]. Current commercially available rigid or flexible endoscopes enable exploration of the gastrointestinal tract, including the esophagus, stomach, colon,

Manuscript received September 23, 2010; revised February 10, 2011; accepted February 12, 2011. Date of publication April 7, 2011; date of current version June 2, 2011. This work was supported in part by the U.S. National Science Foundation under Grant ECCS 0702095, in part by the Wisconsin Institutes for Discovery, and in part by the Wallace H. Coulter Foundation. Subject Editor O. Solgaard.

$\mathrm{X}$. Zeng is with the Department of Electrical and Computer Engineering, University of Wisconsin, Madison, WI 53706 USA (e-mail: xzeng2@ wisc.edu).

C. T. Smith is with the Department of Biomedical Engineering, University of Wisconsin, Madison, WI 53706 USA, and also with the Department of Surgery, University of Wisconsin School of Medicine and Public Health, Madison, WI 53706 USA (e-mail: csmith4@uwhealth.org).

J. C. Gould and C. P. Heise are with Department of Surgery, University of Wisconsin School of Medicine and Public Health, Madison, WI 53706 USA (e-mail: gould@surgery.wisc.edu; heise@ surgery.wisc.edu).

H. Jiang is with the Department of Electrical and Computer Engineering, the Department of Biomedical Engineering, the Materials Science Program and the Eye Research Institute, University of Wisconsin, Madison WI 53706 USA (e-mail: hongrui@engr.wisc.edu).

Color versions of one or more of the figures in this paper are available online at http://ieeexplore.ieee.org.

Digital Object Identifier 10.1109/JMEMS.2011.2127456 and in part the small bowel, as well as the joint spaces, the abdominal cavity, thorax, and other structures. This has aided in understanding their structures and functions and is essential in diagnosing pathology and delivering therapy [2]. In recent years, due to technical improvements, such as the invention of wireless capsule endoscopy [3] and robotic surgery [4], minimally invasive surgery has become a common medical procedure. The future of endoscopy holds promise for advanced minimally invasive procedures like natural orifice transluminal endoscopic surgery.

Current fiber endoscopes use single optical lenses, such as rod lenses and gradient index lenses, at the distal ends of fibers or in front of charged-couple devices (CCDs) for imaging [5], [6]. However, the focal length of these single lenses is nontunable. The tiny lenses in a conventional zoom optical system require supporting rings to hold the bulk of the lenses and actuators to move the lenses in the system; they are hard to manufacture and assemble due to the small space in endoscopes. Resulting from these single lenses, the large depth of focus (DOF) is required for clearer images, but the depth perception in images is lost, which is one of the largest challenges for surgeons to adapt a 2-D flat view of the surgical field [7]. Some 3-D depth recovery has been realized by software estimations but with limited success [8]. Hence, endoscopic surgeons need extensive training due to the need for constant and skillful manual maneuvering of the endoscopes during procedures [2], [7].

Tunable-focus microlenses integrated at the end of fiber endoscopes can scan the areas of interest with minimal or no back-and-forth movements of the scopes themselves. Benefitting from the variable focal length of the microlenses, different DOFs and thus spatial depth perception can be obtained in the images, which would greatly advance endoscopy and increase the efficiency of endoscopic surgeons [9], [10]. Tunable-focus microlenses have been achieved by mechanical pressure [11], [12] or displacement [13], electrochemistry [14], electrical field [15], electrowetting [16], [17], and environmentally adaptive hydrogel [18]. However, current tunable microlens technologies are not best suited for this due to their relatively large size, complicated fabrication, and demanding actuation [19]. In addition, these methods require mechanical, electrical, and environmental signals to tune microlenses; there remains a challenge to integrate these microlenses with other optical components in endoscopes. Particularly in the biological and medical fields, electrical controls and high-pressure fluid circulation should be avoided [20].

We recently reported preliminary results on liquid microlenses actuated through infrared (IR) light-responsive 


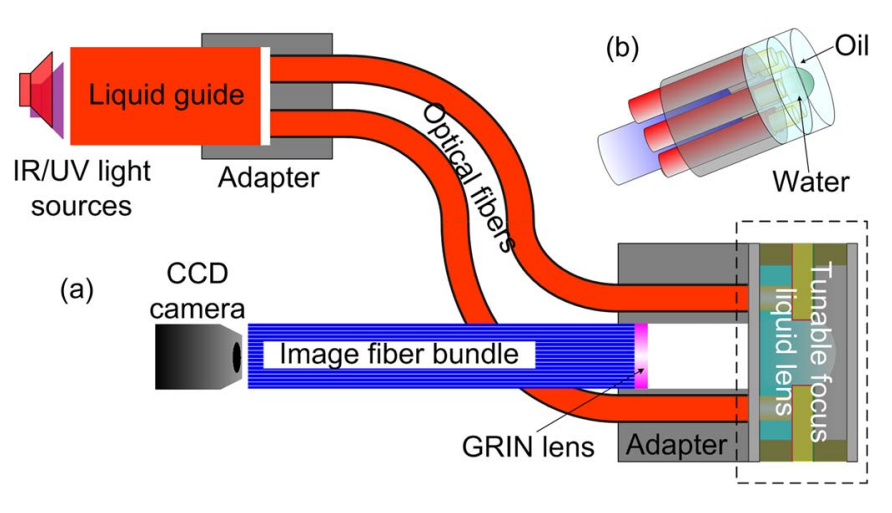

Fig. 1. (a) Schematic and (b) 3-D schematic of a prototype endoscope with a liquid tunable-focus microlens integrated at its end and actuated through IR light. Two sets of optical fibers are used: the actuation fibers and the image acquisition fiber bundle. IR light is transmitted via the actuation of optical fibers to tune the focal length of the microlens at the end of the fibers. Images from the microlens are transferred to a camera via the image acquisition fiber bundle.

hydrogel microstructures [9]. Here, we demonstrate prototype endoscopes with tunable-focus liquid microlenses integrated at their distal end and actuated through IR light. Light transmitted through optical fibers was used for the operation and image acquisition of the microlenses. IR light-responsive hydrogel microstructures, which were made of thermo-responsive $\mathrm{N}$ isopropylacrylamide (NIPAAm) hydrogels with entrapped gold nanoparticles that had distinct and strong absorption at the IR spectrum, were used for the actuation of the microlenses. Two types of endoscopes, i.e., Endoscopes I and II, are presented here. The hydrogel microstructures were defined by fibers in Endoscope I and by masks in Endoscope II. The response time and the alignment of microlenses from these two endoscopes were compared. The focal length of a typical microlens varied from 52 to $8 \mathrm{~mm}$. The angle of view (AOV) of Endoscope II was measured to be from $77^{\circ}$ to $128^{\circ}$. Microlens arrays with various sizes of microlenses could potentially obtain diverse DOF and increase the field of view (FOV).

\section{PRinciples AND Structures}

Fig. 1 shows the (a) schematic and (b) 3-D schematic of a prototype endoscope with a liquid tunable-focus microlens that is integrated at the distal end. Two sets of optical fibers, i.e., actuation optical fibers and image acquisition fiber bundle, are connected to the back side of the microlens. IR light is transmitted via the actuation optical fibers to actuate the hydrogel microstructures and thus vary the focal length of the microlens at the end of the fibers. Images from the tunablefocus microlens are transferred to a camera via the image acquisition fiber bundle.

Fig. 2 shows the cross-sectional structure of the liquid tunable-focus microlens and its two statuses, i.e., divergent and convergent. We previously reported on the mechanism of the microlens [9]. A curved water-oil interfacial meniscus forms the liquid microlens, and its circumference is pinned by a hydrophobic-hydrophilic (H-H) boundary at the top edge of an aperture [9], [18]. Oil prevents the evaporation of water and serves as lens material owing to its higher refractive index (1.48) than water (1.33).

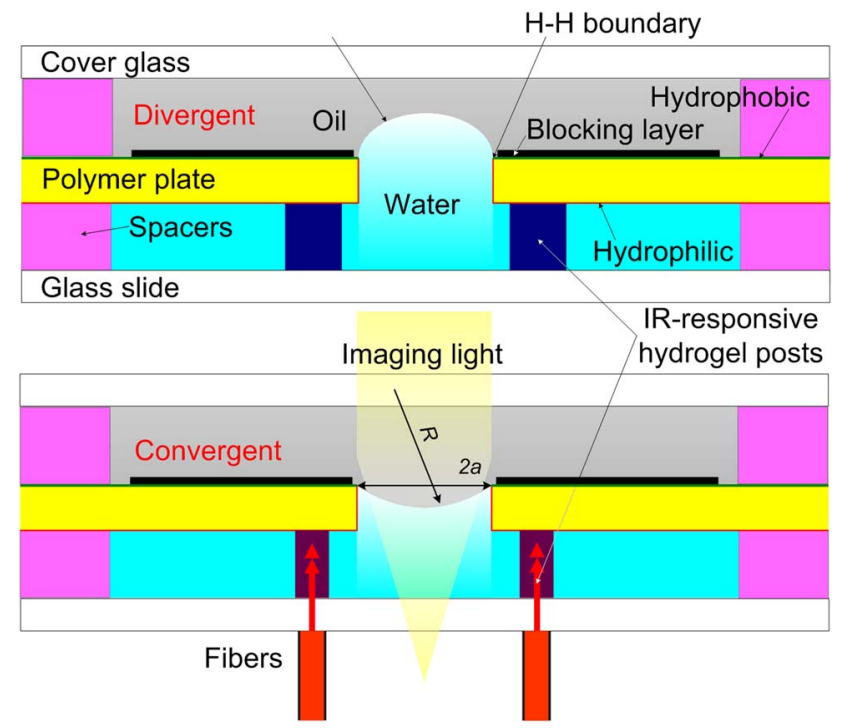

Fig. 2. Schematic of a liquid tunable-focus microlens in two statuses: divergent and convergent. A water container consists of two components: a polymer plate with an aperture for the top plate and a glass slide for the bottom plate. The sidewalls and bottom surfaces of the aperture are chemically treated hydrophilic, whereas the top surfaces are naturally hydrophobic, forming an $\mathrm{H}-\mathrm{H}$ boundary. A curved water-oil interfacial meniscus, pinned by the $\mathrm{H}-\mathrm{H}$ boundary at the top edge of the aperture, forms the microlens. Oil prevents the evaporation of water and serves as lens materials owing to the higher refractive index. The microlens is actuated by multiple IR light-responsive hydrogel microstructures defined either by a photomask or by the actuation optical fibers. Gold nanoparticles are entrapped in the network of the hydrogels and generate heat from the IR light to control the temperature in the hydrogels. The volumetric change in the hydrogels depending on temperature regulates the pressure difference across the water-oil interfacial meniscus, protruding upward (divergent) with the expanded hydrogels and bulging downward (convergent) with the contracted hydrogels, and thus tunes the resultant focal length of the microlens. Under IR light, the microlens scans from far to near, which is called a "forward" cycle. After the IR light is removed, the hydrogels expand back to the original status, and the microlens scans "reversely." In both cycles, the microlens operates in the convergent status.

The microlens is actuated by multiple IR light-responsive hydrogel microstructures, which are formed in the water container under UV radiance. The position of each hydrogel post is defined either by a photomask or by each actuation optical fiber. The polymer chains in the IR light-responsive hydrogel can either repel each other and be swollen or attract each other and be very compact, depending on local temperatures controlled by irradiated IR light, resulting in the volumetric change of the hydrogel [21]. The volumetric change regulates the pressure difference across the water-oil meniscus. The meniscus protrudes upward with an expanded hydrogel and bulges downward with a contracted hydrogel, varying its curvature and the resultant focal length of the microlens (from divergent to convergent). Therefore, the microlens can scan the areas of interest with minimal or no back-and-forth movements and obtain spatial information from different DOFs of the microlens. The IR light-responsive hydrogel contains gold nanoparticles with distinct and strong optical absorption of IR light [9], [22]. Under IR light, the nanoparticles in the hydrogel absorb IR light, generating heat to cause the hydrogel to contract. The microlens thus scans from far to near, which is called a "forward" cycle. After the IR light is removed, heat dissipates, the hydrogel expands back to the original status, and the microlens scans "reversely." In both cycles, the microlens is designed to always operate to converge light. 


\section{FABRICATION PROCESSES}

\section{A. Polymer Materials}

The microlens consists of three polymers: 1) poly isobornyl acrylate (poly-IBA) for the formation of the water container including an aperture and a microchannel; 2) polydimethylsiloxane (PDMS) for the formation of the oil container; and 3) IR light-responsive hydrogel, namely, NIPAAm hydrogel entrapped with gold nanoparticles, for the actuation component of the microlens. Under UV radiation, the IBA and NIPAAm hydrogel solutions can be solidified and photopatterned because of the photopolymerization (like negative photoresist) called liquid-phase photopolymerization or $\mathrm{LP}^{3}$. The detailed recipe of poly-IBA and PDMS can be found in previous articles [9], [23], [24].

The IR light-responsive hydrogel consists of five components: 1) $0.545 \mathrm{-g}$ NIPAAm as the monomer; 2) $0.0385 \mathrm{-g}$ 2,2-dimethoxy-2-phenylace-tophenone as the comonomer; 3) $0.031-\mathrm{g} \mathrm{N}, \mathrm{N}^{\prime}$-methylenebisacrylamide as the cross-linker; 4) $0.75-\mathrm{mL}$ dimethyl sulfoxide; and 5) $0.25-\mathrm{mL}$ gold nanoparticle solution as the solvents. All the chemicals are used as received.

The water-soluble gold nanoparticles coated with thiolated poly-(ethylene glycol) (PEG) ligand are synthesized using Brust method [9], [25], [26]. The thiolated PEG chains are methyl terminated and do not add chemical functionality. Benefitting from this coated polymer, the nanoparticles are aqueous soluble and uniformly entrapped in the polymerized NIPAAm hydrogel network after polymerization. First, $2.19 \mathrm{~g}$ of tetraoctylammonium bromide $\left(\left[\mathrm{CH}_{3}\left(\mathrm{CH}_{2}\right)_{7}\right]_{4} \mathrm{NBr}\right)$ was dissolved in $80 \mathrm{~mL}$ of toluene, and $30 \mathrm{~mL}$ of a $30-\mathrm{mM}$ aqueous hydrogen tetrachloroaurate $\left(\mathrm{HAuCl}_{4}\right)$ solution was added. Under stirring, $100 \mathrm{mg}$ of hexanethiol was added, followed by slowly adding $25 \mathrm{~mL}$ of $0.4-\mathrm{M}$ aqueous sodium borohydride $\left(\mathrm{NaBH}_{4}\right)$ solution. After the mixture was stirred for $1 \mathrm{~h}$, the solvent was evaporated, and the organic layer was isolated. The particles were washed three times with ethanol. The gold particles were then collected and dispersed in $10 \mathrm{~mL}$ of dichloromethane (DCM). Next, $10 \mathrm{~mL}$ of $1.82-\mathrm{mM}$ aqueous polyethylene glycol (PEG) solution was added for the exchange reaction. After $24 \mathrm{~h}$, DCM was removed under reduced pressure with mild heat, and the particles with excessive PEG were redispersed in DCM and stirred for another $48 \mathrm{~h}$. The particles were again evaporated dry, redispersed in deionized (DI) water, and centrifuged to remove insoluble particles. The concentration of gold nanoparticles in the solution was around $4.7 \mathrm{mg} / \mathrm{mL}$. Fig. 3 shows the scanning electron microscope (SEM) image of the synthesized gold nanoparticles coated with PEG-thiol polymer. To take the SEM image, a droplet of gold nanoparticle solution was placed onto a glass slide and evaporated dry. The SEM image was taken with thermally assisted field emission SEM (Leo 1530, Zeiss/LEO, Oberkochen, Germany). The diameter of the gold nanoparticles is between 10 and $20 \mathrm{~nm}$.

\section{B. Device Fabrication}

The device was fabricated through $\mathrm{LP}^{3}$ without the need for a clean room facility [9], [23]. Fig. 4 shows the fabrication

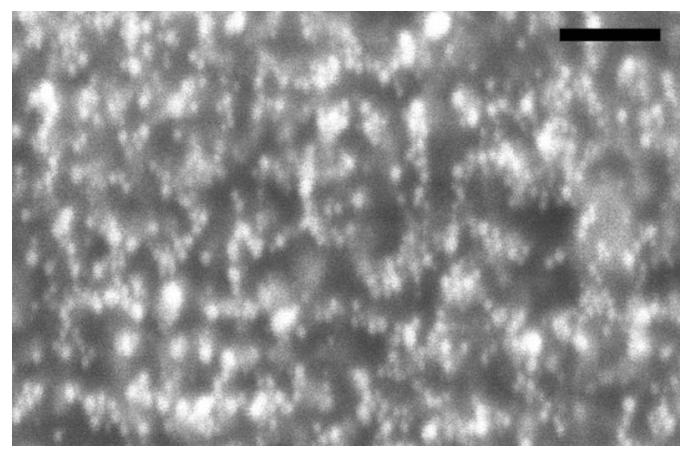

Fig. 3. SEM image of the gold nanoparticles coated with PEG-thiol polymers. To take the SEM image, a droplet of gold nanoparticles solution is placed onto a glass slide and evaporated to dry. The scale bar represents $100 \mathrm{~nm}$.

process flow of a tunable-focus microlens integrated at the ends of optical fibers. First, a polycarbonate cartridge cavity (40 mm $\times 22 \mathrm{~mm}$, HybriWells, Grace Bio-Labs, Inc., Bend, OR, USA) was filled with IBA solution. The thickness of the cavity was defined by $250-\mu \mathrm{m}$-thick spacers (3M Corporate, St. Paul, MN, USA). After filling the chamber with IBA prepolymer solution, a film mask (Mask I) was placed on top of the cartridge to pattern a circular poly-IBA aperture in the cavity through $\mathrm{LP}^{3}$ under UV radiance (intensity $I_{\mathrm{UV}}=8.2 \mathrm{~mW} / \mathrm{cm}^{2}$; time, $t=23 \mathrm{~s}$ ), as shown in Fig. 4(a). The UV radiance was controlled by a desktop EXFO Acticure S2000 (EXFO Photonics Solutions, Inc., Missisauge, ON, Canada). The masks were transparent films with high resolution (3000 dpi) printed by Imagesetter, Inc. (Madison, WI, USA).

Then, the bottom liner plate was peeled off, and the polyIBA aperture plate with the cartridge plate was flipped over. Next, another film mask (Mask II) was used as the top plate, and a cavity around $800 \mu \mathrm{m}$ thick was formed by similar spacers (3M Corporate, St. Paul, MN, USA). A water container and a connecting microchannel were formed on top of the poly-IBA aperture plate through $\mathrm{LP}^{3}$ using the same exposure intensity but the different exposure time $(t=35 \mathrm{~s})$, as shown in Fig. 4(b).

Next, the mask was removed from the top of the water container. The sidewalls and top surfaces of the poly-IBA plate were treated from hydrophobic to hydrophilic with corona discharge plasma for $30 \mathrm{~s}$ [27] (BD-20AC, Electro-Technic Products Inc., Chicago, IL, USA), as shown in Fig. 2(c). The red lines in Fig. 4(c) stand for the hydrophilic surfaces. The contact angle of the DI water on surfaces was measured by a goniometer (OCA 15+, Dataphysics Instruments Inc., Germany). The contact angles of the DI water on the surface of the poly-IBA plate were $105^{\circ}$ and $47^{\circ}$, respectively, before and after plasma treatment. The bottom surfaces maintained their natural hydrophobicity because of the protection from the cartridge plate. Therefore, an H-H boundary was formed at the top edge of the aperture.

Then, the bottom cartridge plate was removed, and the whole poly-IBA plate was flipped over again and bonded onto a glass slide $(76.2 \mathrm{~mm} \times 25.4 \mathrm{~mm} \times 1.0 \mathrm{~mm}$, Fisher Scientific Inc., Pittsburgh, PA, USA), completing the water container and the connecting microchannel, as shown in Fig. 4(d). Epoxy (ITW Devcon, Danvers, MA, USA) was applied to the edge of the plate to prevent the leakage of liquids. An 
(a)

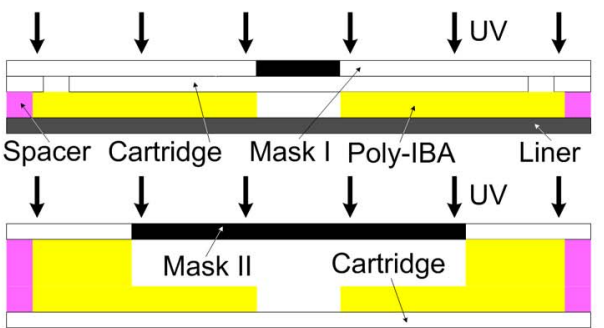

(c)

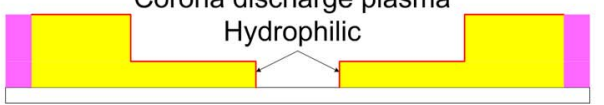

Hydrophobic

(d)

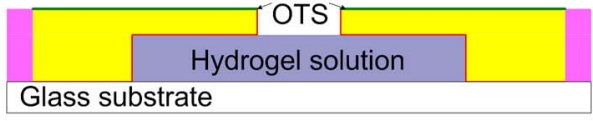

(e I)

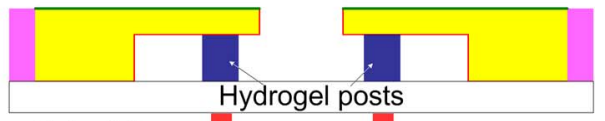

II)

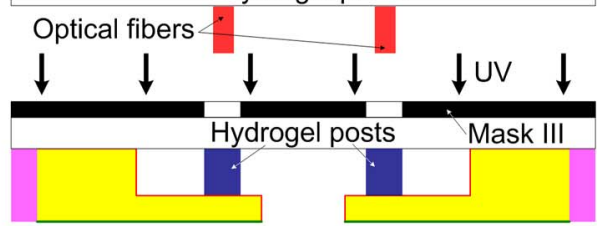

Blocking layer

(f)

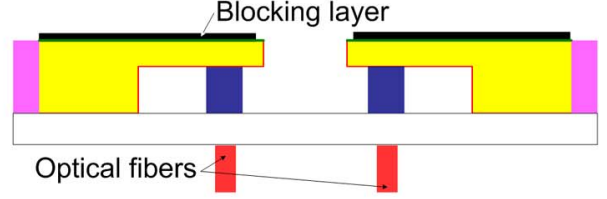

(g)

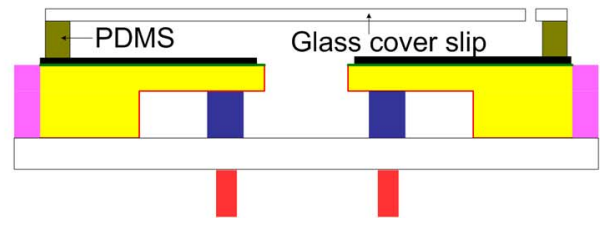

Fig. 4. Fabrication process flow of a tunable-focus liquid microlens integrated at the end of optical fibers. The whole process is based on $\mathrm{LP}^{3}$. (a) A polyIBA aperture is patterned in the cavity under UV radiance. The thickness of the cavity is defined by the spacers and is $250 \mu \mathrm{m}$. (b) The bottom liner plate is peeled off, and the cartridge plate is flipped over. A film mask is used as the top plate, and a cavity around $800 \mu \mathrm{m}$ thick is formed. A water container and a connected microchannel are formed on top of the poly-IBA aperture plate through $\mathrm{LP}^{3}$. (c) The sidewalls and top surfaces of the polyIBA plate are treated from hydrophobic to hydrophilic with corona discharge plasma. (d) The bottom cartridge plate is removed, and the whole poly-IBA plate is flipped over again and is bonded onto a glass slide, forming the water container and the connecting microchannel. The top surface of the aperture plate is treated with OTS for better hydrophobicity. The IR light-responsive hydrogel solution is flowed into the water container through the microchannel. (e I) A set of optical fibers are connected to the back side of the glass slide. Hydrogel microstructures are photopolymerized and patterned in the water container under UV light emitted from the corresponding optical fibers. Each hydrogel post is self-aligned with the corresponding optical fiber. (e II) A mask is placed onto the back side of the glass slide, and hydrogel microstructures are formed in the water container through $\mathrm{LP}^{3}$. Afterwards, the optical fibers are connected to the back side of the glass slide. The hydrogel microstructures number more than the optical fibers. The light emitted from one optical fiber can be absorbed by multiple hydrogel microstructures. (f) A blocking layer made of a nontransparent film is placed on top of the aperture plate to reduce or eliminate the effect of strong IR light on images. (g) A PDMS ring, whose surface is treated with corona discharge plasma to improve the adhesion, is bonded on top of the blocking layer and covered by a glass slide to form an oil chamber.

octadecyltrichlorosilane (OTS) solution diluted by hexadecane $(0.2 \% \mathrm{v} / \mathrm{v})$ was applied onto the top surface of the aperture plate to coat a monolayer for higher hydrophobicity. As a result, the contact angle of the DI water increased to $116^{\circ}$. The green lines in Fig. 4(d) stand for the hydrophobic surfaces. The IR lightresponsive hydrogel solution flowed into the water container through the microchannel.

Two methods were shown here to form the hydrogel microstructures in the water container. Each method is for a different endoscope prototype. The results and the performance of these hydrogel microstructures on the microlenses and endoscopes are compared in Sections V-C and V-E, respectively. Fig. 4(e I) shows the setup to pattern the hydrogel microstructures in the water container by utilizing optical fibers, which was used in Endoscope I. A set of optical fibers $(1035 \mu \mathrm{m}$ cladding, F-MBE; $630 \mu \mathrm{m}$ cladding, F-MSC; Newport Corporation, Irvine, CA, USA) were bound and attached to the back side of the glass slide by machined adapters. Each hydrogel post was patterned under UV light emitted from the corresponding optical fiber for $3 \mathrm{~s}$. The output intensity of UV light from the fiber was measured by a power meter (FieldMaxII laser power meter, Coherent Inc., Santa Clara, CA, USA) with a UV sensor (OP-2 UV enhanced silicon optical sensor, Coherent Inc., Santa Clara, CA, USA) to be $4.8 \mathrm{~mW} / \mathrm{cm}^{2}$.

The other method is to define the hydrogel microstructures by a mask, which was used in Endoscope II. The mask (Mask III) was placed onto the back side of the glass slide, and the UV exposure parameters were $I_{\mathrm{UV}}=12.5 \mathrm{~mW} / \mathrm{cm}^{2}$ and $t=19 \mathrm{~s}$, as shown in Fig. 4(e II). Afterwards, the optical fibers were bound together and attached to the back side of the glass slide. The number of hydrogel microstructures is more than that of optical fibers. The light emitted from one optical fiber could be absorbed by multiple hydrogel microstructures.

Next, a blocking layer made of a nontransparent film was placed on top of the aperture plate to reduce and eliminate the effect of IR light on images in the "forward" cycle, as shown in Fig. 4(f). As shown in Section V-E, without this blocking layer, no images could be taken during the "forward" cycle because the IR light for the actuation made the FOV too bright. Finally, a PDMS ring, whose surface was treated with corona discharge plasma to improve the adhesion, was bonded on top of the blocking layer and covered by a glass slide to form an oil chamber, as shown in Fig. 4(g).

\section{Model of Change in Focal Length}

To estimate the dynamic focal length of microlenses as a function of time, a simple physical model is presented. Here, we ignore the gravity and assume that the water-oil interfacial meniscus is spherical. Thus, the relationship between the volume of water $V$ and the radius of curvature of the water-oil meniscus $R$ is given as

$$
V=\frac{\pi}{3}\left(R-\sqrt{R^{2}-a^{2}}\right)\left(R^{2}+a^{2}-R \sqrt{R^{2}-a^{2}}\right)
$$

where $a$ is the radius of the aperture. 
TABLE I

COMPARISON OF Two TYPES OF ENDOSCOPES: ENDOSCOPES I AND II

\begin{tabular}{|l|l|l|}
\hline & Endoscope I & Endoscope II \\
\hline optical fiber $(\mu \mathrm{m})$ & 600 & 1000 \\
\hline $\begin{array}{l}\text { Number of actuation optical } \\
\text { fiber }\end{array}$ & 6 & 12 \\
\hline $\begin{array}{l}\text { Method of defining hydrogel } \\
\text { microstructures }\end{array}$ & Via actuation optical fibers & Via masks \\
\hline Number of defined hydrogel \\
posts
\end{tabular}

Thus, the focal length of the microlens can be calculated from

$$
f=\frac{n_{\text {water }} \cdot R}{n_{\text {oil }}-n_{\text {water }}}
$$

Here, $n_{\text {oil }}$ and $n_{\text {water }}$ are the refractive indices of oil (1.48) and water (1.33). Then, the calculated curves are fit with the measured points.

\section{EXPERIMENTS AND RESULTS}

\section{A. Two Types of Endoscopes}

Here, we present two types of endoscopes, i.e., Endoscopes I and II. First, Endoscope I was assembled and tested. Based on the testing results of Endoscope I, improvements were made to the design, and Endoscope II was then assembled and tested. The differences between Endoscopes I and II are listed in Table I.

\section{B. Absorption Spectra of Gold Nanoparticle Solution and Hydrogel}

The absorption spectra were measured by an Edmund Industrial Optics CCD spectrometer (Edmund Optics, Barrington, NJ, USA). The scanning wavelength range was from 400 to $1050 \mathrm{~nm}$. All the liquid samples were measured in cuvets with 1-mm-long light path. The hydrogel with entrapped gold nanoparticles was polymerized in a cuvet under UV radiance $\left(I_{\mathrm{UV}}=12.5 \mathrm{~mW} / \mathrm{cm}^{2}\right)$ for $5 \mathrm{~min}$. Fig. 5 shows the absorption spectra of the hydrogel solution, the polymerized hydrogel, DI

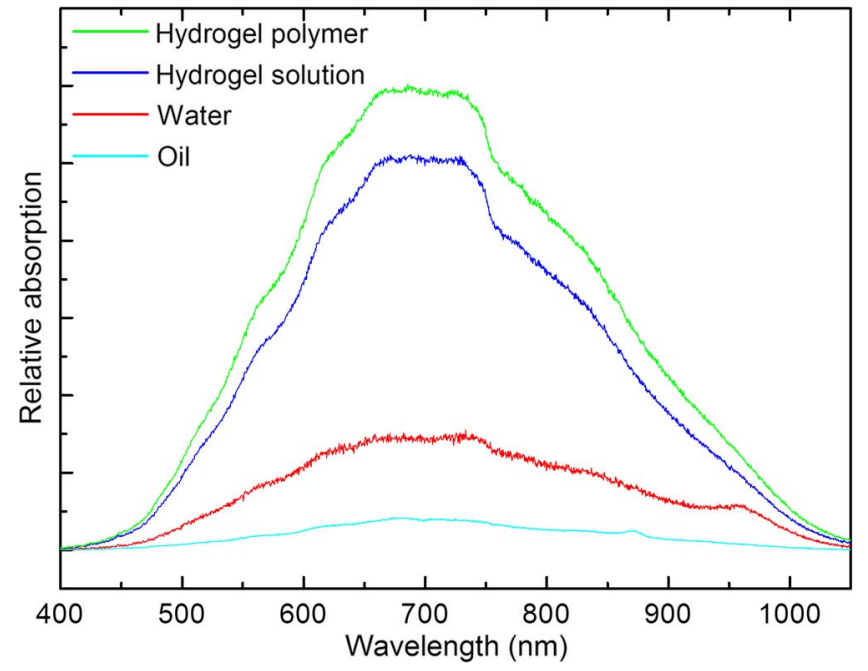

Fig. 5. Absorption spectra of the polymerized IR light-responsive hydrogel with entrapped gold nanoparticles, gold nanoparticle solution, DI water, and oil. The gold nanoparticles in the solution and entrapped in hydrogel have a peak absorption between 660 and $730 \mathrm{~nm}$.

water, and oil. The gold nanoparticles in the hydrogel solution and the polymerized hydrogel had a peak absorption between 660 and $730 \mathrm{~nm}$, which was at near-IR spectrum.

\section{Patterned Hydrogel Microstructures}

Fig. 6 shows a photo of the patterned hydrogel microstructures. The hydrogels were defined either by the actuation optical fibers or by the mask, as described in Section III-B. Two kinds 


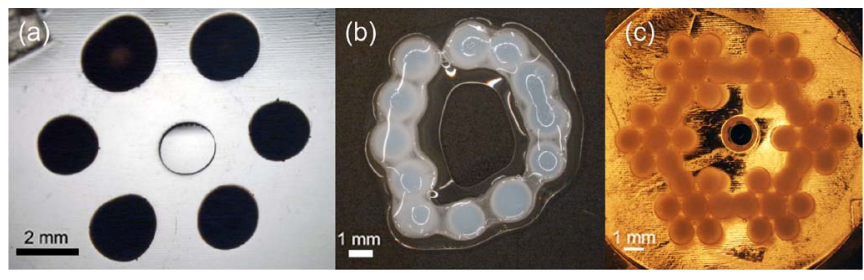

Fig. 6. Photo of the hydrogel microstructures photopatterned in the water container under UV radiance. (a) The hydrogel microstructures are formed under UV light transmitted via six optical fibers whose diameter is $1 \mathrm{~mm}$. (b) The hydrogel microstructures are formed under UV light transmitted via 12 optical fibers whose diameter is $600 \mu \mathrm{m}$. Owing to the large radiation angle of UV light emitted from optical fibers, the patterned hydrogel microstructures are larger than the optical fibers. Because the fibers are cut manually by a fiber cleaver and an incision is left on the cutting surface of the optical fibers, light emitting from the optical fiber and the resultant patterned hydrogel microstructures are not round and uniform in shape. (c) The hydrogel microstructures are formed through Mask III shown in Fig. 4(e II) under UV radiance. The mask-defined hydrogel microstructures are more compact and uniform than the fiber-defined microstructures.

of optical fibers with different sizes were tested, as listed in Table I. In Fig. 6(a), UV light was emitted from six bound optical fibers (F-MBE multimode fiber, Newport Corporation, Irvine, CA, USA) whose core diameter was $1 \mathrm{~mm}$. In Fig. 6(b), the UV light was from 12 bound fibers (F-MSC multimode fiber, Newport Corporation, Irvine, CA, USA), whose core diameter was $600 \mu \mathrm{m}$. Owing to the large radiation angle of the UV light emitted out from the optical fibers, the patterned hydrogel microstructures were larger than the optical fibers. Because the fibers were cut manually by a fiber cleaver (F-CL1, Newport Corporation, Irvine, CA, USA), and an incision was left on the cutting surface of the optical fibers, the light emitting from the optical fibers and the resultant patterned hydrogel microstructures were not round and uniform in shape.

In Fig. 6(c), there were 42 hydrogel microstructures formed under UV radiance via Mask III in Fig. 4(e II). Compared with those hydrogel microstructures defined by fibers, the maskdefined hydrogel microstructures were more compact and uniform but not as well aligned. In addition, without the limitation from machining, more and denser hydrogel microstructures could be formed through masks, and thus, the response time of the microlens could be improved (decreased) [9].

\section{Connecting Adapters}

In Endoscope I, an image acquisition fiber bundle ProVision PV618 (Pro-Vision Video System, Kentwood, MI, USA) was used, whose diameter is $6 \mathrm{~mm}$ and pixel number is 3200 . To increase the resolution and reduce the size of fiber bundles, Zibra Milliscope II S/N-P3919 (Zibra Corporation, Westport, MA, USA), whose diameter is $2 \mathrm{~mm}$ and pixel number is 17 000, was used for transferring images in Endoscope II. All the optical fibers and fiber bundles were bound together by adapters and were attached to the back side of a microlens. Fig. 7 shows a photo of Endoscope II with the tunable-focus microlens integrated at its end. The Zibra fiber bundle and the 12 optical fibers (F-MSC) were used here. There were two sets of adapters used. One set was to connect the liquid guide from the light sources to the actuation optical fibers, and the other set was to connect the actuation optical fibers and the

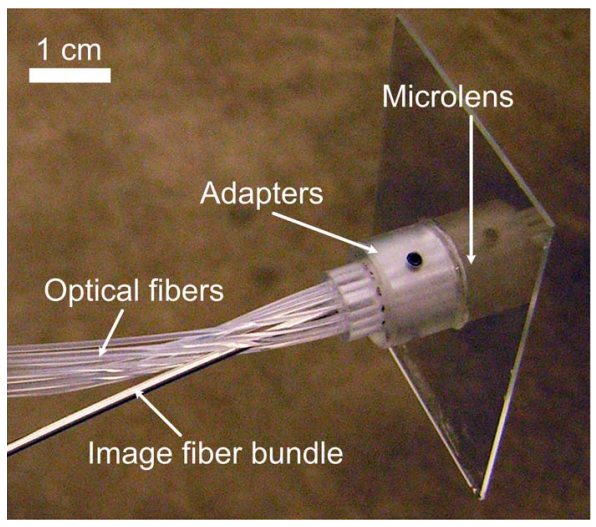

Fig. 7. Photo of Endoscope II with a tunable-focus liquid microlens at their end. An image acquisition fiber bundle (Zibra) and 12 optical fibers (F-MSC) are bound together and attached to the back side of the microlens by adapters.

image acquisition fiber bundle to the back side of the glass slide with the microlens, as shown in Fig. 7. All the adapters were made of aluminum alloy or polycarbonate and were fixed by set screws [28]. The transmission efficiency of IR light from a light source (1000-W research quartz tungsten halogen lamp, Newport Corporation, Irvine, CA, USA) to the hydrogel microstructures was measured to be less than $3 \%$.

\section{E. Images of Objects From Two Endoscopes With Tunable-Focus Microlenses}

Endoscope I consists of a microlens with six hydrogel microstructures formed through the optical fibers, as shown in Fig. 6(a), and the ProVision fiber bundle connecting to a regular camera (Minolta DiMAGE Z1 digital camera). The diameters of the microlens and each hydrogel post were 1.8 and $2 \mathrm{~mm}$, respectively. The IR light used to actuate the hydrogel microstructures was generated from the light source and transmitted via the optical fibers, as shown in Fig. 1. No blocking layer was used in this microlens. A transparent film with the printed logo "W" was placed $50 \mathrm{~mm}$ away from the microlens. Images from the endoscope with the microlens were taken and recorded. Fig. 8(a) shows the frame sequence of the focused images in the "reverse" scanning cycle of the microlens transferred from the ProVision fiber bundle. Each hexagon pixel stands for one fiber in the image acquisition fiber bundle. No images were shown here for the "forward" cycle because without the blocking layer, the IR light for actuation makes the FOV too bright. At the time instant of $0 \mathrm{~s}$, when the IR light was off, the hydrogels were most contracted. Owing to the dissipation of heat, the hydrogels began to expand to the original swollen status. The focal length of the microlens and the size of the resultant image increased. The "forward" and "reverse" scanning cycles took 30 and $35 \mathrm{~s}$, respectively.

For Endoscope II, the microlens with 42 hydrogel microstructures, as shown in Fig. 6(c), formed through the mask was attached to the Zibra fiber bundle. The diameters of the microlens and each hydrogel post were 1.8 and $1 \mathrm{~mm}$, respectively. Two transparent films with the printed $\log _{0}$ "W" and "UW" were placed 15 and $50 \mathrm{~mm}$, respectively, away from the microlens at one end of the fiber bundle. The 

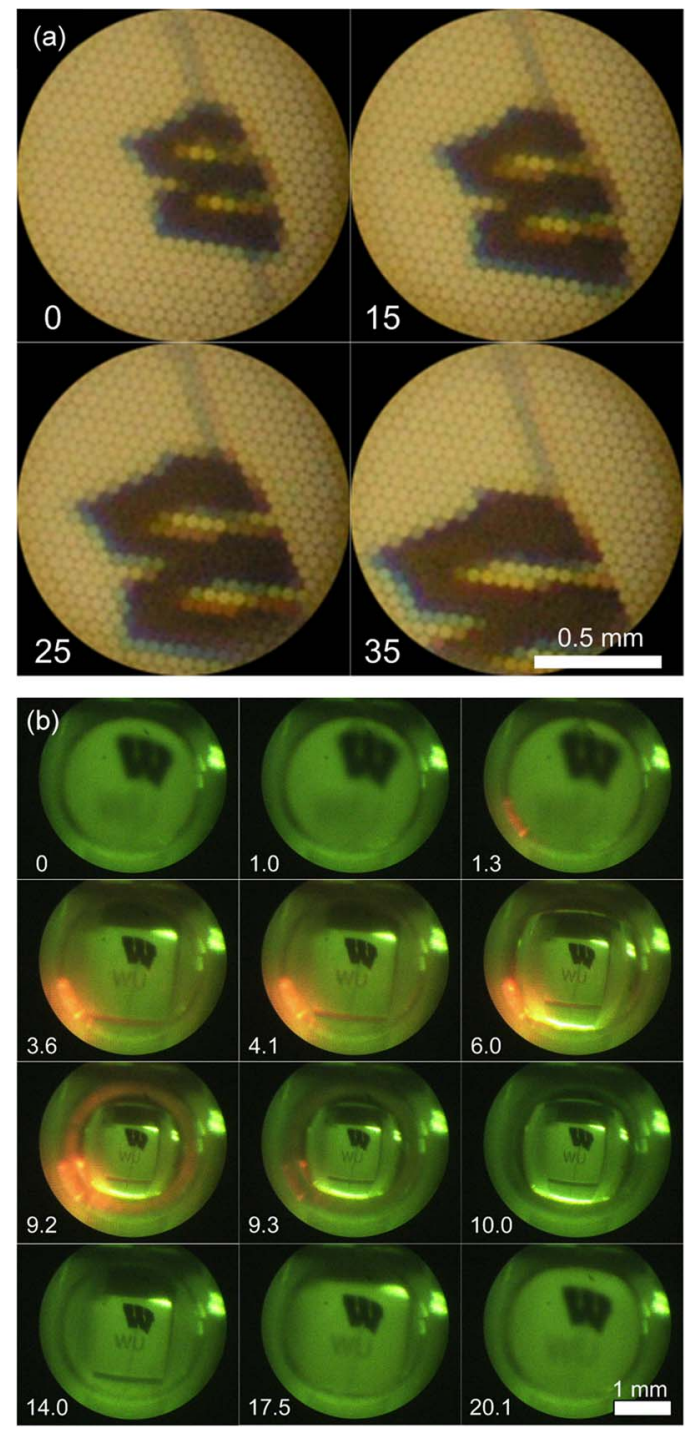

Fig. 8. (a) Frame sequence of the focused images obtained from Endoscope I in the "reverse" scanning cycle of the microlens. Owing to the strong IR light for actuation during the "forward" scanning cycle, the images are blank and are not shown here. The microlens is actuated by six hydrogel microstructures formed through optical fibers. Each hexagon pixel stands for one fiber in the image acquisition fiber bundle. At $0 \mathrm{~s}$, when the IR light is switched off, the hydrogels are most contracted. Owing to the dissipation of heat, the hydrogels begin to expand to the original swollen status. The focal length of the microlens increases, and thus the size of the resultant image increases. (b) Frame sequence of the focused images obtained from Endoscope II in one scanning cycle of the microlens. The microlens is actuated by 42 hydrogel microstructures formed through the photomask. From 1.3 to $9.3 \mathrm{~s}$, the IR light is on, and the microlens scans the area in a "forward" cycle. The logo "W" is focused at $3.6 \mathrm{~s}$. The $\operatorname{logo}$ "UW" is focused at $6.0 \mathrm{~s}$. After $9.3 \mathrm{~s}$, the IR light is off, and the microlens begins to scan "reversely." At 10.0 and 14.0 s, the microlens subsequently scans the two images plates again. Finally, at 20.1 s, the microlenses complete one scanning cycle.

images were recorded by an industrial CCD camera (AVT Stingray IEEE1394 C-mount cameras, Allied Vision Technologies $\mathrm{GmbH}$, Germany) connected to the other end of the fiber bundle. Fig. 8(b) shows the frame sequence of the focused images obtained from the fiber bundle within one scanning cycle. Initially, the images were out of focus and blurry. From the time instant of 1.3-9.3 s, the IR light was on, and the microlens scanned along the optical axis "forwardly." At the time instant of $3.6 \mathrm{~s}$, the image plate with the logo "W" was focused. Then, at the time instant of $6.0 \mathrm{~s}$, the image plate with the logo "UW" was focused. From the time instant of $9.2 \mathrm{~s}$, both image plates were out of focus and became blurry again. After the time instant of $9.3 \mathrm{~s}$, the IR light was off and the microlens began to scan "reversely." At the time instant of 10.0 and $14.0 \mathrm{~s}$, the microlens subsequently scanned the two image plates again. Finally, at the time instant of $20.1 \mathrm{~s}$, the microlens completed the scanning of the area, and both image plates were blurry.

From the device fabrication, the fiber-defined hydrogel microstructures in Endoscope I have better alignment to the optical fibers. However, because the size of the hydrogel microstructures is larger and their number is fewer, the response time of the microlenses actuated by them is slower. On the other hand, many mask-defined hydrogel microstructures can easily and simultaneously be formed with a smaller size, and thus, the response time of microlenses in Endoscope II actuated by them is much shorter. Nonetheless, the alignment between these mask-defined hydrogel microstructures and optical fibers needs to be carefully handled.

\section{F. Images of Simulated Organs From Endoscope II}

To obtain more clinically relevant images, an endoscopic environment was constructed to simulate the human colon and was observed by Endoscope II. The colon was simulated from flexible plastic pipe (Amerimax Home Products Inc., Chicago, IL), and its inner and outer diameters were 95 and $115 \mathrm{~mm}$, respectively. Simulated tissue (Simulab Corporation, Seattle, WA) was used to create colonic polyps with diameter ranging from 3 to $10 \mathrm{~mm}$. This mimics the range of polyps that are clinically relevant, as most are less than $10 \mathrm{~mm}$ [29]. Fig. 9 shows the frame sequence of the focused images of the simulated colon and polyps obtained from Endoscope II in one scanning cycle. Initially, there were three polyps in the field. From the time instant of $1.8 \mathrm{~s}$, the IR light was on, and the FOV began to expand. At the time instant of $4.6 \mathrm{~s}$, there were four polyps in the other end of the simulated colon in the field. From the time instant of $5.2 \mathrm{~s}$, the IR light was off, and the FOV of the microlens began to shrink. At the time instant of $11.4 \mathrm{~s}$, the microlens went back to the original status.

\section{G. Effect of the Size of Microlenses on Focal Length}

The microlens array with three microlenses was simultaneously actuated by 18 hydrogel posts and was used to scan image planes, as shown in Fig. 10(a). The diameters of the microlenses were $1.2,1.5$ and $1.8 \mathrm{~mm}$, respectively, and each hydrogel post was $1 \mathrm{~mm}$ in diameter. Two logos, i.e., $\mathrm{W}$ and $\mathrm{UW}$, were printed on transparency films and were 54 and $104 \mathrm{~mm}$, respectively, below the glass substrate with the microlens array. A camera was placed above the microlens array to monitor and record images. Fig. 10(b) shows the focused image from the microlens array at a certain time. Each microlens focused on different focal planes with a limited DOF. The spatial perception could be obtained by overlaying images from each microlens.

The focal length of the convergent microlens was measured by optically determining the minimum focused point of a collimated incident light beam along the optical axis at 


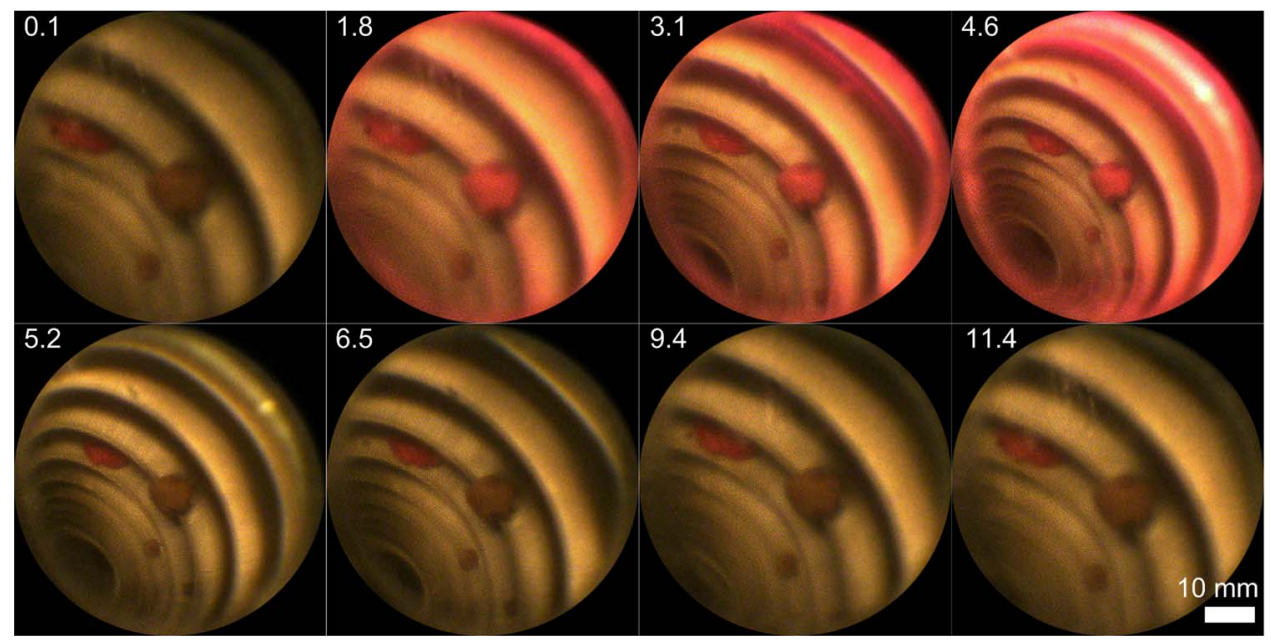

Fig. 9. Frame sequence of the focused images of the simulated human colon and polyps obtained from Endoscope II in one scanning cycle of the microlens. Initially, three polyps could be observed in the field. From the time instant of $1.8 \mathrm{~s}$, the IR light was on, and the AOV began to increase. At the time instant of $4.6 \mathrm{~s}$, one extra polyp and the other end of the simulated colon lumen could be observed in the field. From the time instant of $5.2 \mathrm{~s}$, the IR light was off, and the $\mathrm{AOV}$ of the microlens began to decrease. At the time instant of $11.4 \mathrm{~s}$, the microlens went back to the original status.

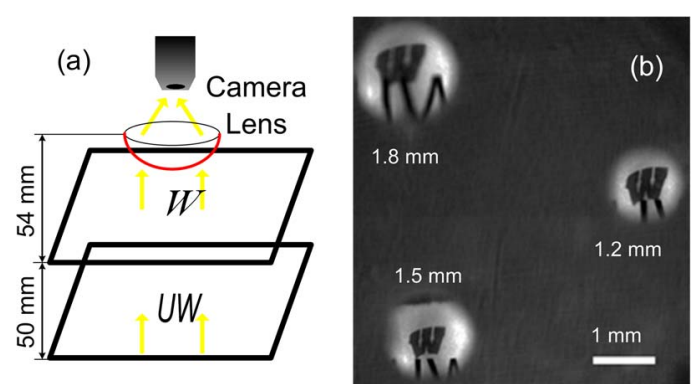

Fig. 10. (a) Schematic of scanning image planes using the tunable-focus microlens array. Two transparency films with the logo "W" and "UW" are located below the microlens array at 54 and $104 \mathrm{~mm}$, respectively. A camera is placed above the microlens array to monitor and record images. (b) Focused image from the microlens array at one time instant showing different focused points. Three microlenses are simultaneously actuated, and their diameters are $1.2,1.5$ and $1.8 \mathrm{~mm}$, respectively. Each microlens focuses on different focal planes with a limited DOF. Therefore, the spatial perception could be obtained by overlaying images from these microlenses.

different temperatures [23]. The temperature variation in the hydrogels caused by IR light irradiation during the microlens operation was recorded by a thermocouple (5SC-TT-K-40-36, Omega Engineering, Inc., Stamford, Connecticut, USA). The dynamic change in the focal length of a microlens array in the "forward" scanning cycle was therefore obtained [9]. Fig. 11(a) shows the schematic setup to measure the focal length of these microlenses. The IR light for the actuation was shone from an oblique angle instead of via the optical fibers in this experiment. The focal length of each microlens was measured separately. The focal length of these microlenses as a function of time was measured and plotted in Fig. 11(b). Error bars indicating the standard deviation in the focal length of the microlens are also shown. The focal length of the microlenses varied from 52 to $8 \mathrm{~mm}$ in half a minute. The curves calculated from (1) and (2) were fit with the measured data.

From (1) and (2), the focal length of the microlenses depends on the size of the apertures. Therefore, multiple microlenses with different sizes of apertures can focus on points at different distances with limited DOF at a given time instant. The DOF of the microlenses depends on the resolution of the optical fibers behind the microlens and could not be unfortunately measured because of the relatively low resolution of the fibers. Each DOF and the corresponding spatial depth perception from each microlens are overlaid, and a much larger DOF of the microlens array can simultaneously be obtained. Microlens arrays integrated at the end of the fiber endoscopes could potentially be utilized to improve the DOF of the images.

\section{H. FOV of Microlenses}

Because of the round images obtained from endoscopes, the AOV decides the range of FOV of the endoscopes. Here, the AOV of Endoscope II was measured. A ruler with scale was placed $3.8 \mathrm{~mm}$ away from the microlens, and the images from Endoscope II were recorded. The AOV of the endoscope was triangularly calculated from the recorded images. The focal length was measured with time, as described before. Table II lists the relationship between the focal length and the AOV of Endoscope II. As we can see, at the shortest focal length, the AOV of Endoscope could reach $128^{\circ}$. However, the image quality at the edge was poor, and the image was severely distorted.

The FOV of endoscopes could further be expanded by utilizing multiple microlenses at the end of endoscopes. Fig. 12 shows the frame sequence of the focused images from a microlens array with multiple microlenses during one scanning cycle. There are seven microlenses with the same size of apertures in this array, which are simultaneously actuated by the hydrogel microstructures. The FOV of each microlens is the same, but their locations are different. Thus, the images from each microlens are different, as shown in the image at the time instant of $18 \mathrm{~s}$ in Fig. 12. The images from each microlens can be overlapped to expand the FOV of the microlens array.

\section{CONCLUSION}

In summary, we have demonstrated prototype endoscopes integrated with tunable-focus liquid microlenses at their distal 

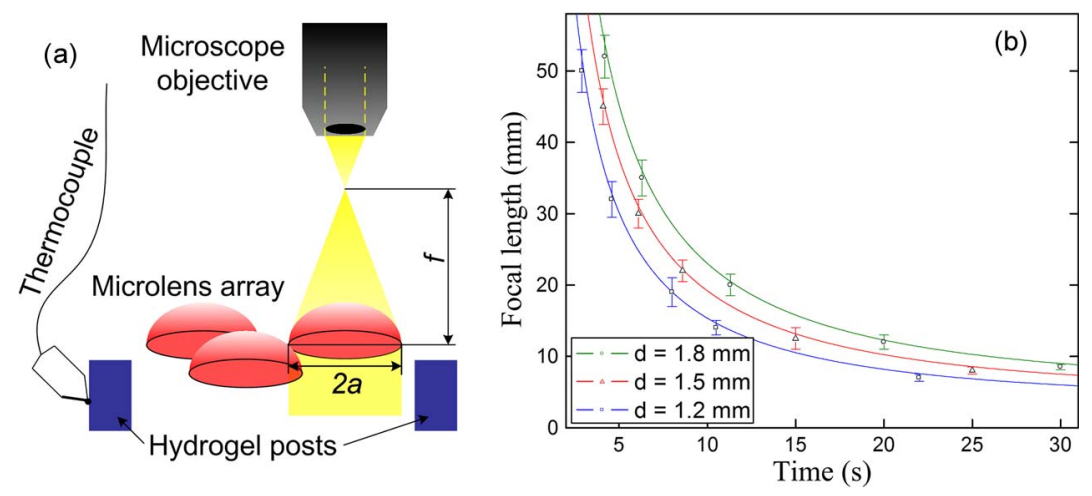

Fig. 11. (a) Schematic of the focal length measurement setup. A collimated light is shone from the bottom of a microlens. The focal point of the microlens is determined as the minimum spot along the optical axis. The distance between the focal point and the microlens is the focal length of the microlens. When the microlens is operated, the temperature variation in the hydrogel caused by IR light irradiation is recorded by a thermocouple. Therefore, the dynamic focal length in the convergent microlens is obtained. (b) Dynamic change in the focal length of a microlens array with three microlenses in the "forward" scanning cycle as a function of time. Error bars indicate the standard deviation in the focal length of the microlens. The focal length range of the microlenses is from $\sim 8$ to $52 \mathrm{~mm}$.

TABLE II

RELATIONSHiP BETWEEN THE FocAl LENGTH AND THE AOV OF ENDOSCOPE II

\begin{tabular}{|l|l|}
\hline Focal length $(\mathrm{mm})$ & Angle of view \\
\hline 52 & $77^{\circ}$ \\
\hline 35 & $92^{\circ}$ \\
\hline 20 & $116^{\circ}$ \\
\hline 12 & $120^{\circ}$ \\
\hline 9 & $128^{\circ}$ \\
\hline
\end{tabular}

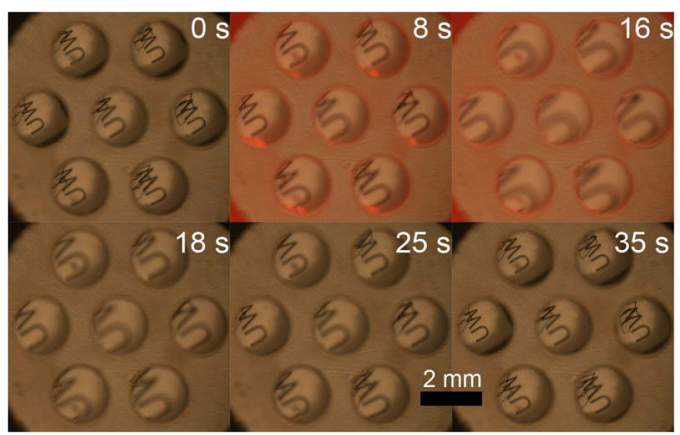

Fig. 12. Frame sequence of focused images from a microlens array with seven microlenses in one scanning cycle. The microlenses with the same size are simultaneously actuated by hydrogel microstructures. Because of the different locations of each microlens, the FOV of the microlens array can be expanded by overlapping the images from each microlens.

ends that are actuated through IR light. Tunable-focus microlenses can not only scan the areas of interest with minimal back-and-forth movements of the scopes themselves but also obtain different DOFs and thus spatial depth perception, which could greatly assist endoscopic surgeons. A liquid microlens is formed by the interface of water and oil and is pinned by an $\mathrm{H}-\mathrm{H}$ boundary at the top edge of a lens aperture. Gold nanoparticles in the hydrogel absorb IR light and convert it to heat. The volumetric change in hydrogel microstructures responding to heat converted from IR light regulates the curvature of the water-oil interface and thus varies the focal length of the microlens. Light for the operation of the microlens and the image acquisition is transmitted via optical fibers. Two fabrication methods of hydrogel microstructures were tested. Compared with the fiberdefined hydrogel microstructures, the mask-defined hydrogel microstructures can easily be formed denser and smaller and thus have faster response time. However, they cannot be selfaligned to the optical fibers. The focal length of a typical microlens was measured to be from 52 to $8 \mathrm{~mm}$. The AOV of Endoscope II varied from $77^{\circ}$ to $128^{\circ}$. A microlens array could potentially be utilized to simultaneously obtain different DOFs of images and thus spatial depth perception. In addition, the FOV could also be expanded by microlens arrays.

In future studies, the size of the endoscope could be decreased by using a smaller image fiber bundle, and the response time of microlenses can be improved by increasing the number of optical fibers and using more hydrogel microstructures with smaller sizes. By utilizing a greater number of fibers, the resolution of images will improve, and the feature size could be as small as micrometers. We also plan to test the endoscopes in an animal model to prove safety prior to any testing in human subjects. Currently, the microlenses are continuously tuned by the accumulation or dissipation of heat. In the future, we are exploring the balance between the accumulation and dissipation of heat and expecting that the focal point could be user controlled by diverse methods, such as microheaters and temperature sensors, pulse-width-modulated IR light, or digitalized actuation by characterizing the volumetric change of each hydrogel microstructures [30]. These user-controlled microlenses could track the movement of tissues and compensate for it by tuning the focal length. Spherically arranged microlens arrays can be fabricated at the end of optical fibers for endoscopy, and a larger DOF and FOV could be achieved [31], [32]. In addition to the application of this optical technology to provide assistance in surgery, tunable-focus microlenses may also be used for optical coherence tomography [33], [34] and scanning confocal optical microscope [35], [36].

\section{ACKNOWLEDGMENT}

The authors would like to thank the Mechanical Engineering Machine Shop, UW-Madison, for optical adapters, R. K. Noll at the Materials Science Center for his assistance in taking SEM images, Prof. L. McCaughan and his student C. Staus for discussions, and C. Li, D. Zhu, and H. J. Cho for discussions and help. This research utilized NSF-supported shared facilities at the University of Wisconsin, Madison. 


\section{REFERENCES}

[1] R. M. Salmore, "Our heritage: A history of gastroenterology and gastroenterology nursing," Gastroenterol. Nursing, vol. 21, no. 2, pp. 40-43, Mar./Apr. 1998.

[2] P. B. Cotton, C. B. Williams, R. H. Hawes, and B. P. Saunders, Practical Gastrointestinal Endoscopy: The Fundamentals, 6th ed. Chichester, U.K.: Willey-Blackwell, 2008.

[3] A. Moglia, A. Menciassi, M. O. Schurr, and P. Dario, "Wireless capsule endoscopy: From diagnostic devices to multipurpose robotic systems," Biomed. Microdevices, vol. 9, no. 2, pp. 235-243, Apr. 2007.

[4] D. Oleynikov, "Robotic surgery," Surg. Clinics North Amer., vol. 88, no. 5, pp. 1121-1130, Oct. 2008

[5] P. R. Schauer, S. Ikramuddin, and J. D. Luketich, "Minilaparoscopy," Semin. Laparoscopic Surg., vol. 6, no. 1, pp. 21-31, Mar. 1999.

[6] J. Knittel, L. Schnieder, G. Buess, B. Messerschmidt, and T. Possner, "Endoscope-compatible confocal microscope using a gradient index-lens system," Opt. Commun., vol. 188, no. 5/6, pp. 267-273, Feb. 15, 2001.

[7] S. B. Bhayani and G. L. Andriole, "Three-dimensional (3D) vision: Does it improve laparoscopic skills? An assessment of a 3D headmounted visualization system," Rev. Urology, vol. 7, no. 4, pp. 211-214, Fall 2005.

[8] D. Stoyanov, A. Darzi, and G. Z. Yang, "A practical approach towards accurate dense 3D depth recovery for robotic laparoscopic surgery," Comput. Aided Surg., vol. 10, no. 4, pp. 199-208, Jul. 2005.

[9] X. Zeng and H. Jiang, "Tunable liquid microlens actuated by infrared light-responsive hydrogel," Appl. Phys. Lett., vol. 93, no. 15, art. no. 151101, Oct. 13, 2008.

[10] J. Duparré, F. Wippermann, P. Dannberg, and A. Bräuer, "Artificial compound eye zoom camera," Bioinspiration Biomimetics, vol. 3, no. 4, art. no. 046008 , Dec. 2008.

[11] K. H. Jeong, G. L. Liu, N. Chronis, and L. P. Lee, "Tunable microdoublet lens array," Opt. Exp., vol. 12, no. 11, pp. 2494-2500, May 31, 2004.

[12] D. Y. Zhang, V. Lien, Y. Berdichevsky, J. Choi, and Y. H. Lo, "Fluidic adaptive lens with high focal length tunability," Appl. Phys. Lett., vol. 82, no. 19, pp. 3171-3172, May 12, 2003.

[13] H. Ren and S. T. Wu, "Variable-focus liquid lens by changing aperture," Appl. Phys. Lett., vol. 86, no. 21, art. no. 211107, May 23, 2005.

[14] C. A. Lopez, C. C. Lee, and A. H. Hirsa, "Electrochemically activated adaptive liquid lens," Appl. Phys. Lett., vol. 87, no. 13, art. no. 134102, Sep. 2005.

[15] N. Binh-Khiem, K. Matsumoto, and I. Shimoyama, "Polymer thin film deposited on liquid for varifocal encapsulated liquid lenses," Appl. Phys. Lett., vol. 93, no. 12, art. no. 124101, Sep. 22, 2008.

[16] S. Kuiper and B. H. W. Hendriks, "Variable-focus liquid lens for miniature cameras," Appl. Phys. Lett., vol. 85, no. 7, pp. 1128-1130, Aug. 2004.

[17] S. Yang, T. N. Krupenkin, P. Mach, and E. A. Chandross, "Tunable and latchable liquid microlens with photopolymerizable components," $A d v$. Mater, vol. 15, no. 11, pp. 940-943, Jun. 5, 2003.

[18] L. Dong, A. K. Agarwal, D. J. Beebe, and H. Jiang, "Adaptive liquid microlenses activated by stimuli-responsive hydrogels," Nature, vol. 442, no. 7102, pp. 551-554, Aug. 2006.

[19] H. Jiang and L. Dong, "Smart lenses," Phys. World, vol. 19, no. 11, pp. 29-31, Nov. 2006.

[20] D. J. Beebe, J. S. Moore, J. M. Bauer, Q. Yu, R. H. Liu, C. Devadoss, and B. H. Jo, "Functional hydrogel structures for autonomous flow control inside microfluidic channels," Nature, vol. 404, no. 6778, pp. 588-590, Apr. 6, 2000.

[21] Y. Osada, J. P. Gong, and Y. Tanaka, "Polymer gels (Reprinted from Functional Monomers and Polymers, pg 497-528, 1997)," J. Macromolecular Sci.-Polymer Rev., vol. C44, no. 1, pp. 87-112, Feb. 2004.

[22] S. R. Sershen, G. A. Mensing, M. Ng, N. J. Halas, D. J. Beebe, and J. L. West, "Independent optical control of microfluidic valves formed from optomechanically responsive nanocomposite hydrogels," $A d v$. Mater, vol. 17, no. 11, pp. 1366-1368, Jun. 6, 2005.

[23] X. Zeng and H. Jiang, "Polydimethylsiloxane microlens arrays fabricated through liquid-phase photopolymerization and molding," J. Microelectromech. Syst., vol. 17, no. 5, pp. 1210-1217, Oct. 2008.

[24] D. J. Beebe, J. S. Moore, Q. Yu, R. H. Liu, M. L. Kraft, B. H. Jo, and C. Devadoss, "Microfluidic tectonics: A comprehensive construction platform for microfluidic systems," Proc. Nat. Acad. Sci. U.S.A., vol. 97, no. 25 , pp. $13488-13493$, Dec. 2000.

[25] M. Brust, M. Walker, D. Bethell, D. J. Schiffrin, and R. Whyman, "Synthesis of thiol-derivatized gold nanoparticles in a 2-phase liquid-liquid system," J. Chem. Soc.-Chem. Commun., no. 7, pp. 801-802, Apr. 7, 1994.

[26] A. H. Latham and M. E. Williams, "Versatile routes toward functional, water-soluble nanoparticles via trifluoroethylester-PEG-thiol ligands," Langmuir, vol. 22, no. 9, pp. 4319-4326, Apr. 25, 2006.
[27] S. Thorslund and F. Nikolajeff, "Instant oxidation of closed microchannels," J. Micromech. Microeng., vol. 17, no. 4, pp. N16-N21, Apr. 2007.

[28] X. Zeng, Microlenses and Their Application in Endoscopes. Madison, WI: Dept. Elect. Comput. Eng., Univ. Wisconsin-Madison, 2009.

[29] M. L. Corman, Colon and Rectal Surgery, 5th ed. Philadelphia, PA: Lippincott Williams \& Wilkins, 2005.

[30] D. W. Lee and Y. -H. Cho, "4-bit digital liquid lens for variable focal length," in Proc. 15th Int. Conf. Solid-State Sens., Actuators, Microsyst., Denver, CO, 2009, pp. 2306-2309.

[31] D. Zhu, C. Li, X. Zeng, and H. Jiang, "Tunable-focus microlens arrays on curved surfaces," Appl. Phys. Lett., vol. 96, no. 8, art. no. 081111, Feb. 22, 2010.

[32] X. Zeng, C. Li, D. Zhu, H. J. Cho, and H. Jiang, "Tunable microlens arrays actuated by various thermo-responsive hydrogel structures," J. Micromech. Microeng., vol. 20, no. 11, art. no. 115035, Nov. 2010.

[33] K. Aljasem, A. Werber, A. Seifert, and H. Zappe, "Fiber optic tunable probe for endoscopic optical coherence tomography," J. Opt. A-Pure Appl. Opt., vol. 10, no. 4, art. no. 044012, Apr. 2008

[34] X. M. Liu, M. J. Cobb, Y. C. Chen, M. B. Kimmey, and X. D. Li, "Rapid-scanning forward-imaging miniature endoscope for real-time optical coherence tomography," Opt. Lett., vol. 29, no. 15, pp. 1763-1765, Aug. 1, 2004

[35] K. Kumar, R. Avritscher, Y. M. Wang, N. Lane, D. C. Madoff, T. K. Yu, J. W. Uhr, and X. J. Zhang, "Handheld histology-equivalent sectioning laser-scanning confocal optical microscope for interventional imaging," Biomed. Microdevices, vol. 12, no. 2, pp. 223-233, Apr. 2010.

[36] A. Jain and H. K. Xie, "Microendoscopic confocal imaging probe based on an LVD microlens scanner," IEEE J. Sel. Topics Quantum Electron., vol. 13, no. 2, pp. 228-234, Mar./Apr. 2007.

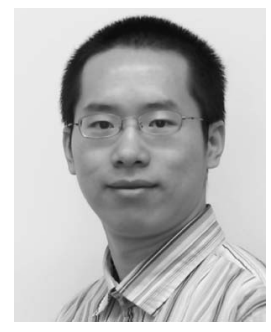

Xuefeng Zeng received the B.S. degree in microelectronic technology from Tianjin University, Tianjin, China, in 2002, the M.S. degree in electronic science and technology from Tsinghua University, Beijing, China, in 2005, and the M.S. and Ph.D. degrees in electrical engineering from the University of Wisconsin, Madison, in 2007 and 2009, respectively.

$\mathrm{He}$ is currently a Postdoctoral Research Associate in the Department of Electrical and Computer Engineering, University of Wisconsin, Madison. His research interests include micro-/nanofabrication technology and devices, microoptics, microfluidics, biomedical sensors, smart materials, and biomimetics and bioinspiration.

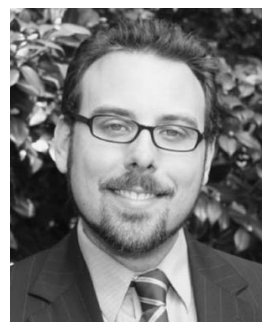

Carter T. Smith received the B.S. degree in electrical and computer engineering from Baylor University, Waco, TX, in 2003, and the M.D. degree from The University of Texas Health Science CenterHouston, Houston, TX, in 2007. He is currently working toward the M.S. degree in biomedical engineering at the University of Wisconsin, Madison.

$\mathrm{He}$ is currently a General Surgery Resident at the University of Wisconsin Hospital and Clinics, Madison. His research interests include medical device development, electronic health records safety and implementation, and advanced optics for diagnosis and staging or rectal cancer. 


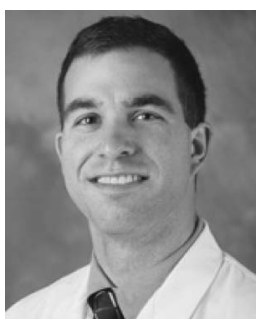

Jon C. Gould received the M.D. degree from the University of Wisconsin Medical School, Madison, in 1996, and completed his General Surgery Residency at the University of Wisconsin Hospital and Clinics, Madison, in 2001, and his Fellowship in minimally invasive surgery at Ohio State University, Columbus, in 2002.

$\mathrm{He}$ is currently an Associate Professor in the Department of Surgery, Division of General Surgery, University of Wisconsin School of Medicine and Public Health. He is certified by the American Board of Surgery. He specializes in minimally invasive laparoscopic surgery of the foregut (stomach and esophagus). In addition, he uses advanced minimally invasive techniques to treat acid reflux, obesity, achalasia, and hernias. His research interests include obesity surgery and surgical education.

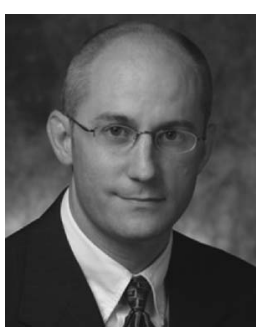

Charles P. Heise received the M.D. degree from the University of Minnesota Medical School, Minneapolis, and completed his General Surgery Residency at the University of Wisconsin Hospital and Clinics, Madison, and his Fellowship in colon and rectal surgery at the University of Minnesota, Minneapolis.

$\mathrm{He}$ is currently an Associate Professor in the Department of Surgery, Division of General Surgery, University of Wisconsin School of Medicine and Public Health. He is certified by the American Board of Colon and Rectal Surgery and by the American Board of Surgery. His specialties include the surgical treatment of colorectal cancer and inflammatory bowel disease. He performs laparoscopic bowel surgery for benign and malignant diseases. His research interests include treatment of diverticulitis and patient outcome and function after surgical treatment of colorectal cancer and inflammatory bowel disease.

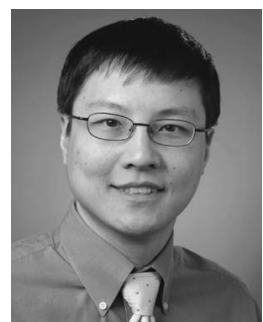

Hongrui Jiang (S'98-M'02-SM'10) received the B.S. degree in physics from Peking University, Beijing, China, and the M.S. and Ph.D. degrees in electrical engineering from Cornell University, Ithaca, NY, in 1999 and 2001, respectively.

From 2001 to 2002, he was a Postdoctoral Researcher at the Berkeley Sensor and Actuator Center, University of California-Berkeley, Berkeley. He is currently an Associate Professor in the Department of Electrical and Computer Engineering, a Faculty Affiliate in the Department of Biomedical Engineering, and a Faculty Member of the Materials Science Program and the Eye Research Institute, University of Wisconsin (UW), Madison. His research interests are in microfabrication technology, biological and chemical microsensors, microactuators, optical MEMS, smart materials and micro-/ nanostructures, lab on a chip, and biomimetics and bioinspiration.

Dr. Jiang received the NSF CAREER Award and the DARPA Young Faculty Award in 2008, and the H. I. Romnes Faculty Fellowship of the University of Wisconsin, Madison, in 2011. 